\title{
Formación y alfabetización del profesor de ELE: de la competencia lingüística a la tecnológica
}

\author{
Eva Álvarez Ramosi®i \\ Universidad de Valladolid, Valladolid, España \\ Leyre Alejaldre Bielii \\ Columbia University, Nueva York, Estados Unidos de América \\ Belén Mateos Blancoiii(i) \\ Universidad de Valladolid, Valladolid, España
}

\begin{abstract}
Resumen
Con el presente trabajo se pretende poner de manifiesto las carencias en formación digital que poseen los másteres de profesorado de lenguas extranjeras, más concretamente de la enseñanza de español como lengua extranjera. Se ha llevado a cabo una revisión bibliográfica que cimente el marco teórico en el que se asienta esta investigación de carácter mixto. Su escasa presencia pone de manifiesto la necesidad de reforzar y ampliar la alfabetización digital en la formación de profesorado y reducir la fractura desigualitaria que la separa del aprendizaje lingüístico. Merece por derecho un tratamiento más profundo y una posición más visible en los planes de estudio.
\end{abstract}

\section{Palabras clave}

Formación docente. ELE. Competencia digital docente. Alfabetización digital.

Formação e alfabetização de professores da ELE: da competência linguagem à competência tecnológica

\begin{abstract}
Resumo
Este artigo tem como objetivo destacar as deficiências no treinamento digital que os professores de mestrado de línguas estrangeiras têm, mais especificamente no ensino de espanhol como língua estrangeira. Foi realizada uma revisão bibliográfica que sustenta o referencial teórico no qual esta pesquisa mista se baseia. Sua baixa presença destaca a necessidade de fortalecer e expandir a alfabetização digital na formação de professores e reduzir a diferença de desigualdade que a separa da aprendizagem de línguas. Merece, por direito, um tratamento mais profundo e uma posição mais visível nos planos de estudo.
\end{abstract}

\section{Palavras-chave}

Formação de professores. ELE. Competência em ensino digital. Alfabetização digital. 


\title{
Professional development and literacy for Spanish/FL instructors: from linguistic to digital competence
}

\begin{abstract}
This paper aims to highlight the deficiencies in digital literacy education within official master's programs for Spanish as a foreign language teachers in Spain. A thorough literature review has been carried out that supports the theoretical framework in which this mixed research is based. Its low presence in the syllabi highlights the need to strengthen and expand digital literacy in professional development courses for Spanish as a foreign language teachers and to reduce the curriculum content gap that separates it from linguistic literacy. Digital competence deserves a deeper treatment and a more visible position in current study plans.
\end{abstract}

\section{Keywords}

Teacher training. SFL. Digital teaching competence. Digital competence.

\section{Introducción}

La presencia cotidiana de las herramientas digitales ha revolucionado el paradigma educativo en las últimas décadas. Estudiantes y profesores se encuentran ante una realidad digitalizada que conlleva grandes ventajas, pero también destapa carencias formativas tanto en la competencia digital docente como en la del aprendiente. Conceptos como "nativos digitales", "alfabetización digital" y "competencia digital docente" se posicionan en el centro de este trabajo, que trata de encontrar las razones por las que existe una brecha formativa entre la competencia lingüística del profesor de español como lengua extranjera (ELE) y su alfabetización digital.

La búsqueda de respuestas comienza describiendo el marco teórico que sostiene este trabajo. Para ello, se expone una revisión bibliográfica que facilite la comprensión de los conceptos mencionados previamente en el marco de las competencias docentes del profesor de ELE, seguidamente se analiza la oferta formativa de posgrado para profesores de ELE y la posición de la competencia digital en sus planes curriculares para poder determinar si la demanda actual de profesores con una alta alfabetización digital se alinea a los contenidos académicos de los cursos examinados. Concluimos con una serie de recomendaciones para asegurar el desarrollo continuo de la competencia digital docente. 


\section{Marco teórico}

La práctica docente en el ámbito de la enseñanza y aprendizaje de ELE se ha ido transformando con la incorporación de las herramientas digitales. La incursión de las tecnologías de la información y el conocimiento (TIC), sigla que se usa mayormente en el contexto didáctico y "[...] que privilegia un enfoque instrumental" (MÉNDEZ SANTOS; PANO ALAMÁN, 2019, p. 5), aparece de forma recurrente en los documentos formativos destinados a describir la competencia digital docente de los profesores de segundas lenguas. Con el objetivo de añadir el componente social y relacional, aparecen las tecnologías de la relación, la información y la comunicación (TRIC) (GABELAS BARROSO; MARTA-LAZO; ARANDA, 2012; MARTA-LAZO; GABELAS, 2016), que incluyen la interacción social y cultural en el marco de las herramientas digitales (GARCÍA NAVARRO; FERNÁNDEZ MUNÍN, 2017) y "[...] la interacción, (co)creación, reflexión $y$, en definitiva, humanismo digital que debe impregnar el proceso educomunicativo en Red" (MARTA-LAZO, 2018, s.p.). La aparición de diferentes siglas, que conviven y tratan de describir la presencia de las herramientas digitales y su impacto en el proceso de enseñanza y aprendizaje, demuestra la complejidad que subyace de la inclusión de recursos digitales en los proyectos curriculares y la necesidad de una formación inicial del profesorado de ELE sólida en lo que se refiere a su uso, aplicación y explotación en el aula. Las TIC y las TRIC conviven ahora con otras siglas que tratan de complementar ese enfoque e incluir otras variables propias del ámbito pedagógico. Así es como surgieron las tecnologías del aprendizaje y el conocimiento (TAC), que se centran en el impacto de las herramientas digitales en la educación, o el término acuñado por Reig (2012), que aúna las herramientas digitales y el ámbito de las ciencias sociales, nos referimos a las tecnologías para el empoderamiento y la participación (TEP), cuyo objetivo es la implementación de los recursos tecnológicos para promover la conciencia crítica, la colaboración, la autonomía y la habilidad de un individuo para cambiarse a sí mismo o sus circunstancias (ROMÁN-MENDOZA, 2018).

Esta amalgama de siglas representa el constante crecimiento de la esfera en la que nos encontramos y cómo las demandas concretas y la evolución terminológica influyen en la descripción de las herramientas digitales según los objetivos que se 
quieran lograr: promover la socialización digital, el empoderamiento, o crear una experiencia de aprendizaje dinámica, realista y duradera. En la revisión bibliográfica, se observa un uso extensivo del acrónimo TIC para hacer referencia a las herramientas digitales, por lo que en las siguientes líneas lo utilizaremos para referirnos de forma general a la utilización de los recursos digitales en la educación universitaria y su relación con la competencia digital docente.

La incorporación efectiva de las herramientas digitales en el proceso de enseñanza es una de las tareas más complicadas, ya que depende de muchos factores, como las infraestructuras del centro, la competencia digital del docente y del aprendiente, el liderazgo académico y de las políticas de centro que apoyen la digitalización y la trasformación de la enseñanza, entre otros.

En las últimas décadas, han surgido políticas que promueven la transformación digital de la educación y que han propiciado la creación de una serie de investigaciones y documentos, en continúa revisión y adaptación, que tratan de describir las competencias digitales de los docentes y los aprendientes para promover un aprendizaje significativo, activo y duradero. Son muchos los documentos oficiales, de diferentes países, que se han publicado sobre el estado de la cuestión, por lo que a continuación presentaremos estudios académicos y documentos oficiales que consideramos más relevantes para definir y acotar qué entendemos por competencia digital docente en el contexto universitario.

La Comisión Europea y los Estados miembros publicaron en 2007 el documento Competencias clave para el aprendizaje permanente: un marco de referencia europeo (COMISIÓN EUROPEA, 2007), basado en la recomendación 2006/962/CE del Parlamento Europeo y del Consejo, de 18 de diciembre de 2006, sobre las competencias clave para el aprendizaje permanente de los ciudadanos. En el documento, se identifican ocho competencias clave (COMISIÓN EUROPEA, 2007): 1. comunicación en la lengua materna; 2. comunicación en lenguas extranjeras; 3. competencia matemática y competencias básicas en ciencia y tecnología; 4. competencia digital; 5. aprender a aprender; 6. competencias sociales y cívicas; 7. sentido de la iniciativa y espíritu de empresa; y 8. conciencia y expresión culturales.

Nos encontramos una de las primeras conceptualizaciones sobre el término competencia digital en un documento europeo: 


\begin{abstract}
La competencia digital entraña el uso seguro y crítico de las tecnologías de la sociedad de la información (TSI) para el trabajo, el ocio y la comunicación. Se sustenta en las competencias básicas en materia de TIC: el uso de ordenadores para obtener, evaluar, almacenar, producir, presentar e intercambiar información, y comunicarse y participar en redes de colaboración a través de Internet. (COMISIÓN EUROPEA, 2007, p. 7).
\end{abstract}

Aunque este texto no se centra en la competencia digital docente, los conocimientos, capacidades y actitudes esenciales se alinean con la docencia y han de extrapolarse a ese ámbito. El docente ha de conocer la naturaleza, función y las oportunidades que ofrecen las herramientas digitales. Para ello, tiene que ser capaz de usar diferentes aplicaciones informáticas, comprender los riesgos y oportunidades de internet, valorar la fiabilidad de los contenidos a los que accede, saber comunicarse en el ámbito digital y utilizar estas herramientas para favorecer la creatividad, el pensamiento crítico y el aprendizaje activo (COMISIÓN EUROPEA, 2007).

Un año después, se publicó el Marco de competencias de los docentes en materia de TIC de la Unesco (2008), que detalla, por un lado, los estándares de la competencia TIC de los docentes y, por otro, presenta orientaciones dirigidas a todos los profesores; directrices para diseñar programas de formación del profesorado y selección de cursos que contribuyen a prepararlos para desempeñar un papel esencial en la capacitación y formación tecnológica de los estudiantes; este documento se actualizó en 2011 y en 2018 (aunque su publicación fue en 2019). En 2013 se publica el DigComp: A Framework for Developing and Understanding Digital Competence in Europe (FERRARI, 2013), con el objetivo de concretar e identificar los componentes clave de la competencia digital en términos de los conocimientos, habilidades y actitudes necesarias para ser competente digitalmente. Este texto propone una serie de descriptores de competencia digital que puedan ser validados a nivel europeo siguiendo los niveles A, B y C. El informe detalla diversos aspectos relacionados con la competencia digital mediante la identificación y enumeración de 21 competencias, organizadas en cinco áreas: Información, Comunicación, Creación de Contenido, Seguridad y Resolución de Problemas. Este marco competencial se convierte en la base del primer Marco Común de Competencia Digital Docente (2013) (MCCDD), que se actualizó en 2017 y que es un documento de referencia en Europa para evaluar la competencia digital docente. En la 
siguiente tabla se muestran las cinco áreas competenciales y las 21 competencias que facilitan la evaluación de la competencia digital docente.

Tabla 1 - Áreas y competencias del MCCDD

\begin{tabular}{|c|c|}
\hline \multirow{3}{*}{$\begin{array}{l}\text { Área 1. Información y } \\
\text { alfabetización } \\
\text { informacional }\end{array}$} & $\begin{array}{l}\text { Competencia 1.1. Navegación, búsqueda y filtrado de información, datos } \\
\text { y contenidos digitales }\end{array}$ \\
\hline & Competencia 1.2. Evaluación de información, datos y contenidos digitales \\
\hline & $\begin{array}{l}\text { Competencia 1.3. Almacenamiento y recuperación de información, datos } \\
\text { y contenidos digitales }\end{array}$ \\
\hline \multirow{6}{*}{$\begin{array}{l}\text { Área 2. Comunicación y } \\
\text { colaboración }\end{array}$} & Competencia 2.1. Interacción mediante las tecnologías digitales \\
\hline & Competencia 2.2. Compartir información y contenidos digitales \\
\hline & Competencia 2.3. Participación ciudadana en línea \\
\hline & Competencia 2.4. Colaboración mediante canales digitales \\
\hline & Competencia 2.5. Netiqueta \\
\hline & Competencia 2.6. Gestión de la identidad digital \\
\hline \multirow{4}{*}{$\begin{array}{l}\text { Área 3. Creación de } \\
\text { contenidos digitales }\end{array}$} & Competencia 3.1. Desarrollo de contenidos digitales \\
\hline & Competencia 3.2. Integración y reelaboración de contenidos digitales \\
\hline & Competencia 3.3. Derechos de autor y licencias \\
\hline & Competencia 3.4. Programación \\
\hline \multirow{4}{*}{ Área 4. Seguridad } & Competencia 4.1. Protección de dispositivos \\
\hline & Competencia 4.2. Protección de datos personales e identidad digital \\
\hline & Competencia 4.3. Protección de la salud \\
\hline & Competencia 4.4. Protección del entorno \\
\hline \multirow{4}{*}{$\begin{array}{l}\text { Área } 5 \text {. Resolución de } \\
\text { problemas }\end{array}$} & Competencia 5.1. Resolución de problemas técnicos \\
\hline & Competencia 5.2. Identificación de necesidades y respuestas tecnológicas \\
\hline & Competencia 5.3. Innovación y uso de la tecnología digital de forma creativa \\
\hline & Competencia 5.4. Identificación de lagunas en la competencia digital \\
\hline
\end{tabular}

Fuente: Elaboración propia a partir de INTEF (2017b).

Esta propuesta surge con "[...] la intención de ofrecer una referencia descriptiva que pueda servir con fines de formación y en procesos de evaluación y acreditación" (INTEF, 2017a, p. 6). Aunque este instrumento no se ha creado para una etapa educativa concreta, consideramos que es aplicable a diferentes niveles formativos, no obstante la investigación de Prendes, Porlán y Sánchez (2018) sostiene que es necesario crear un instrumento adecuado al nivel de docencia, ya que tras una extensa revisión bibliográfica sobre la competencia digital docente en la que se examinaron trabajos publicados a partir del año 2000 y entre los que se destacan las publicaciones de Fernández (2001), Olcott y Schmidt (2002), Hanna (2002), Badia (2004), Mishra y Koehler (2006, 2008), Oliveira, Cervera y Martí (2009), Gallego, Gámiz y Gutiérrez (2010), Oliveira (2010), Pozos (2010), Prendes (2010), Gisbert y Esteve (2011), Gutiérrez (2011), Krumsvik (2011), Carrera y Coiduras (2012), Prendes y Gutiérrez (2013), Durán, Sánchez y Prendes (2016a, 2016b), Prendes, Porlán y Sánchez (2018), Durán, Prendes y Sánchez (2019) y Alejaldre Biel y Álvarez Ramos (2019). Fueron muy pocos los que se situaban en el contexto universitario (PRENDES; PORLÁN; SÁNCHEZ, 
2018; DURÁN; PRENDES; GUTIÉRREZ, 2019) y entre ellos destacan la propuesta de Pozos (2010), el trabajo de Carrera y Coiduras (2012), Mengual y Roig (2012) y Mengual, Roig y Blasco (2016), la extensa investigación de Prendes (2010) y el trabajo de Alejaldre y Álvarez (2019).

Otra reflexión muy interesante que plantea la investigación de Durán, Prendes y Guttiérez (2019, p. 192) es que "[...] la mayoría de los trabajos de investigación sobre competencia digital se centran en instrumentos de autopercepción del profesorado sobre sus niveles de competencia y trabajos sobre el uso de las TIC"1. Esto implica que los estudios presentan instrumentos de medida de la competencia digital docente que se basan principalmente en la autopercepción que tiene el docente sobre sus habilidades digitales, mientras que la propuesta de Durán, Prendes y Gutiérrez (2019) se centra en la creación de una prueba de certificación de la competencia digital del profesorado universitario, y no en una herramienta de autopercepción.

A pesar de las múltiples publicaciones en torno a las habilidades que el docente ha de desempeñar con las herramientas digitales, todavía no existe una definición consensuada para definir la competencia digital docente, por lo que presentaremos en las siguientes líneas las más relevantes.

Tejada (2009, p. 12) destaca la transformación que las TIC han supuesto en el rol del profesor y sostiene que "[...] se tendrá que abogar por el dominio de nuevas competencias profesionales que garanticen tanto el saber, como el saber hacer, el saber estar y el saber hacer en y con TIC", lo que implica que un docente del siglo XXI debe saber implementar las herramientas digitales de forma efectiva en el proceso de enseñanza y aprendizaje. Para lograr esas destrezas, es preciso que exista una formación en alfabetización digital. Teniendo en cuenta lo recién mencionado, Krumsvik (2011, p. 44) define la competencia digital como:

[...] la competencia del profesor/formador de profesores en el uso de las TIC en un contexto profesional con buen criterio pedagógico-didáctico y su conciencia de sus implicaciones para las estrategias de aprendizaje y la formación digital de los alumnos y estudiantes.

1 Citan aquí a autores como Prendes (2010); Prendes, Castañeda y Gutiérrez (2010); Gisbert, Espuny y González (2011); Roig y Pascual (2012); Marcelo, Yot y Mayor (2015); Gutiérrez y Serrano (2016); Boudet (2017); Hatlevik (2017); Gudmundsdottir y Hatlevic (2018); Tourón et al. (2018).

Educ. Form., Fortaleza, v. 6, n. 1, e3521, jan./abr. 2021

DOI: https://doi.org/10.25053/redufor.v6i1.3521

https://revistas.uece.br/index.php/redufor/index ISSN: 2448-3583 
Cada definición aporta algo innovador, globalizador e integrador, así ocurre con la propuesta de Carrera y Coiduras (2012, p. 15), que consideran que esa competencia incluye:

[...] conocimientos, capacidades, actitudes y estrategias que [...] el profesor o el educador debe ser capaz de activar, adoptar y gestionar en situaciones reales para facilitar el aprendizaje alcanzando mayores niveles de logro y promover procesos de mejora e innovación permanente en los procesos formativos.

Finalmente es fundamental señalar que la competencia digital de los docentes de ELE no se limita a saber cómo usar un abanico de herramientas digitales y saber desenvolverse en el mundo virtual, sino que implica el desarrollo de conocimientos y capacidades que permitan entender, desarrollar y aplicar las cinco áreas competenciales del Marco Común de Competencia Digital Docente².

El interés de la comunidad educativa por definir la competencia digital docente junto a la búsqueda de una herramienta de certificación de dicha competencia muestra la necesidad de continuar la investigación para poder ofrecer herramientas válidas que contribuyan a determinar si existe una brecha entre la alfabetización académica del docente de ELE y su alfabetización digital. En el siguiente epígrafe, con el objetivo de determinar de forma cuantitativa la distribución de los contenidos curriculares en los planes curriculares de 30 másteres de profesorado de ELE y poder evaluar si existe un equilibrio entre las materias meramente lingüísticas y las relacionadas con el desarrollo de la competencia digital docente, describimos de forma cuantitativa la distribución de los contenidos curriculares de los másteres estudiados.

\section{Metodología}

Nos encontramos ante una investigación no interactiva de corte analítico, cuyo objetivo es determinar si existe una brecha formativa entre la alfabetización académica de los profesores de ELE y su competencia digital.

En el marco del presente estudio y con el objetivo de establecer de forma cuantitativa la distribución de los contenidos curriculares en los planes de estudio de los másteres de profesorado de ELE y poder evaluar si existe un equilibrio entre las materias

2 Algunas de las experiencias docentes estudiadas así lo demuestra, véase, por ejemplo, Álvarez (2017b), al mostrar las fases y la problemática a la hora de crear una plataforma virtual de ELE.

Educ. Form., Fortaleza, v. 6, n. 1, e3521, jan./abr. 2021

DOI: https://doi.org/10.25053/redufor.v6i1.3521

https://revistas.uece.br/index.php/redufor/index ISSN: 2448-3583 
meramente lingüísticas y las relacionadas con el desarrollo de la competencia digital docente, hemos implementado una variedad de herramientas investigadoras, tanto de corte cuantitativo como cualitativo, que nos han permitido presentar el estado de la cuestión, la recogida de datos cualitativos y su posterior análisis.

La revisión bibliográfica se erige como los cimientos del marco teórico que sostienen esta investigación. A través del estudio de una gran variedad de textos académicos y publicaciones oficiales, se aclaran conceptos clave para poder comprender qué es la competencia digital docente y de qué herramientas disponemos para evaluarla y acreditarla. Seguidamente y con la ayuda de herramientas cuantitativas, analizamos los contenidos curriculares de 30 másteres de formación de profesores de ELE para poder ponderar la posición que ocupa en la formación de posgrado la competencia digital docente.

En lo que concierne a la técnica de recolección de datos y obligados por la tipología de la investigación, hemos optado por el análisis documental, eligiendo como instrumento la ficha de registro de datos. Para la obtención de los mismos, se ha tenido en cuenta la oferta educativa disponible en universidades españolas, tomando solamente aquellas muestras correspondientes a títulos de máster oficial, sin tener presente los títulos propios. Tampoco han formado parte del estudio las organizaciones educativas no universitarias, como institutos de estudios académicos o centros adscritos.

Una vez descritas y analizadas las características formativas de estos másteres, se procederá a presentar los resultados y finalmente se propondrá una serie de recomendaciones para asegurar una posición relevante a las asignaturas de corte tecnológico en los planes educativos de másteres de ELE.

\section{Resultados}

Comenzamos con el análisis de contenidos curriculares prestando atención a la configuración de los planes de estudio de la muestra analizada. Hay tres factores clave que inciden en la formación tecnológica docente: la carga de sistema europeo de transferencia y acumulación de créditos (ECTS), la tipología de la asignatura y su contenido. 
La mayoría de ellos, siguiendo la normativa recogida en el Real Decreto 1393/2007, de 29 de octubre, por el que se establece la ordenación de las enseñanzas universitarias oficiales (art. 15.2), exige la superación de 60 créditos ECTS ${ }^{3}$. Cada uno de ellos se distribuye entre materias optativas y materias obligatorias.

No en todos los planes de estudio comparados se oferta formación en competencia digital docente. Resulta bastante revelador - Tabla 2 - que un $27 \%$ no consideren oportuna la alfabetización digital; más si tenemos presente todo lo vertido en apartados anteriores, donde se ha demostrado que la competencia digital docente engloba mucho más contenido que el uso de internet o la utilización de herramientas digitales. Las áreas y competencias recogidas en el MCCDD constatan esta afirmación. La carencia, por tanto, es bastante significativa y se verá incrementada por los datos que mostraremos a continuación.

Tabla 2 - Másteres sin formación en competencia digital docente

\begin{tabular}{|c|c|}
\hline Máster & Universidad \\
\hline $\begin{array}{c}\text { Máster Universitario en Enseñanza de Español como } \\
\text { Lengua Extranjera }\end{array}$ & Universidad de Cantabria \\
\hline $\begin{array}{c}\text { Máster Universitario Online en Enseñanza de } \\
\text { Español como Lengua Extranjera }\end{array}$ & Universidad de Burgos \\
\hline $\begin{array}{c}\text { Máster Universitario en Español y su Cultura: } \\
\text { Desarrollos Profesionales y Empresariales }\end{array}$ & Universidad de Las Palmas de Gran Canaria \\
\hline $\begin{array}{c}\text { Formación del Profesorado de Español como Lengua } \\
\text { Extranjera }\end{array}$ & Universidad Católica San Carlos de Murcia \\
\hline $\begin{array}{c}\text { Máster Universitario en Enseñanza de Español como } \\
\text { Lengua Extranjera (ELE) }\end{array}$ & Universidad Internacional de La Rioja \\
\hline $\begin{array}{c}\text { Máster Universitario en Lengua Española y Literatura: } \\
\text { Investigación y Aplicaciones Profesionales }\end{array}$ & Universidad de Jaén \\
\hline $\begin{array}{c}\text { Español como Lengua Extranjera: Enseñanza e } \\
\text { Investigación }\end{array}$ & Universidad de Valladolid \\
\hline $\begin{array}{c}\text { Máster de Español como Lengua Extranjera en } \\
\text { Ambitos Profesionales }\end{array}$ & Universidad de Barcelona \\
\hline
\end{tabular}

Fuente: Elaboración propia (2020).

Dentro de los másteres que sí recogen en su plan docente la formación digital hay que separar los que lo hacen como materia obligatoria (algo más de un tercio) Tabla 3 -, lo que conlleva una percepción superior de la necesidad de consecución de

3 Con un número superior de créditos, se encuentra la Universidad de Educación a Distancia (65 ECTS), la Universidad de Navarra (75 ECTS) y las Universidades de León y de Barcelona (90 ECTS).

Educ. Form., Fortaleza, v. 6, n. 1, e3521, jan./abr. 2021

DOI: https://doi.org/10.25053/redufor.v6i1.3521

https://revistas.uece.br/index.php/redufor/index 
la destreza, de aquellos en los que es opcional. La optatividad no viene más que reforzar ese vacío dentro de los programas formativos de capacitación digital docente.

Tabla 3 - Asignaturas obligatorias ofertadas

\begin{tabular}{|c|c|c|}
\hline Universidad & Asignatura & ECTS \\
\hline Universidad de Barcelona & Las TIC en la Didáctica de ELE & 5 \\
\hline Universidad Carlos III & Las TIC en la Enseñanza de ELE & 5 \\
\hline Francisco de Vitoria & Las Nuevas Tecnologías en la Clase de ELE & 4 \\
\hline Universidad de Navarra & $\begin{array}{c}\text { Nuevas Tecnologías Aplicadas a la Enseñanza de } \\
\text { Español }\end{array}$ & 6 \\
\hline Universidad Pablo de Olavide & $\begin{array}{c}\text { Aplicación de Nuevas Tecnologías al Aprendizaje } \\
\text { del Español como L2 }\end{array}$ & 3 \\
\hline Universitat Rovira I Virgili & $\begin{array}{l}\text { Nuevas Tecnologías Aplicadas a la Enseñanza de } \\
\text { ELE }\end{array}$ & 3 \\
\hline Universidad a Distancia de Madrid & $\begin{array}{c}\text { Tecnologías de la Información y Comunicación y } \\
\text { la Enseñanza del Español como Lengua } \\
\text { Extranjera }\end{array}$ & 6 \\
\hline $\begin{array}{l}\text { Universidad Internacional } \\
\text { Menéndez Pelayo }\end{array}$ & $\begin{array}{c}\text { Tecnologías de la Información y la Comunicación } \\
\text { Aplicadas a la Enseñanza del Español }\end{array}$ & 1 \\
\hline Universitat d'Alacant & $\begin{array}{c}\text { Nuevas Tecnologías de la Información y la } \\
\text { Comunicación en la Enseñanza de Segundas } \\
\text { Lenguas/Lenguas Extranjeras }\end{array}$ & 6 \\
\hline Universidad Nebrija & $\begin{array}{c}\text { Entornos Virtuales de Aprendizaje y Recursos } \\
\text { Digitales }\end{array}$ & 3 \\
\hline Universidad Internacional de Valencia & e-Learning 2.0 y 3.0 en el Aula de ELE & 3 \\
\hline
\end{tabular}

Fuente: Elaboración propia (2020).

Un número equivalente de másteres - Tabla 4 - oferta formación de carácter optativo, con un número de créditos (entre tres y seis) semejante a la del tipo obligatorio - Tabla 4. En este caso, la problemática deviene de la elección o el rechazo del discente. Es decir, la formación mínima puede efectuarse o no dependiendo de por lo que opte el alumno de máster.

Tabla 4 - Asignaturas optativas ofertadas

\begin{tabular}{|c|c|c|}
\hline Universidad & Asignatura & ECTS \\
\hline Universidad de Granada & $\begin{array}{c}\text { Tecnologías de la Información y la } \\
\text { Comunicación: Aplicaciones y Usos }\end{array}$ & 3 \\
\hline Universidad de León & $\begin{array}{c}\text { Nuevas Tecnologías Aplicadas a la Enseñanza } \\
\text { de ELE }\end{array}$ & 3 \\
\hline Universidad de Sevilla & $\begin{array}{c}\text { Las TIC en la Clase de ELE. Medios } \\
\text { Audiovisuales y Aula Virtual }\end{array}$ & 4 \\
\hline Universidad de Córdoba & $\begin{array}{c}\text { Español para Fines Específicos y Nuevas } \\
\text { Tecnologías en ELE }\end{array}$ & 4 \\
\hline $\begin{array}{c}\text { Universidad Nacional de Educación a } \\
\text { Distancia }\end{array}$ & $\begin{array}{c}\text { Las Tecnologías de la Información en la } \\
\text { Enseñanza del Español L2 }\end{array}$ & 5 \\
\hline Universidad de Extremadura & $\begin{array}{c}\text { Nuevas Tecnologías en la Enseñanza de ELE } \\
\text { Universidad de Oviedo }\end{array}$ & $\begin{array}{c}\text { Tecnologías de la Información y Comunicación } \\
\text { Aplicadas a la Enseñanza de E/LE }\end{array}$ \\
\hline Universidad de Salamanca & $\begin{array}{c}\text { Nuevas Tecnologías en la Enseñanza de } \\
\text { Segundas Lenguas }\end{array}$ & 3 \\
\hline Universidad Complutense & Las TIC en la Enseñanza de Español como & 6 \\
\hline
\end{tabular}

Educ. Form., Fortaleza, v. 6, n. 1, e3521, jan./abr. 2021

DOI: https://doi.org/10.25053/redufor.v6i1.3521

https://revistas.uece.br/index.php/redufor/index 


\begin{tabular}{|c|c|c|}
\hline & Lengua Extranjera & \\
\hline Universidad Nebrija & TIC Aplicadas a la Enseñanza de ELE & \\
\hline
\end{tabular}

Fuente: Elaboración propia (2020).

Mención aparte merecen el máster impartido en la Universidad de Alcalá, que hasta el curso académico 2018/2019 ofertaba la asignatura optativa, de cinco créditos ECTS, Recursos Tecnológicos para la Enseñanza de la Lengua Española y sus Literaturas, pero que la ha eliminado del plan de estudios en las dos últimas convocatorias, y la configuración bipartita que le confiere la Universidad de Córdoba, al compartir espacio en una única asignatura con una materia (fines específicos) que guarda poca o ninguna relación con la alfabetización tecnológica. Estos dos hechos vienen a exhibir la poca atención y el mínimo valor que se le da a la adquisición de competencias digitales en el ámbito de la formación de profesores.

La media de asignaturas totales matriculadas por máster se sitúa entre 12 y 20, ofertándose solamente una materia para el desarrollo de la destreza, lo que supone apenas una media del $7 \%$ (un máximo del $8.5 \%$ y un mínimo del $5 \%$ ). El equilibrio entre el aprendizaje lingüístico y el tecnológico se ve claramente desestabilizado hacia el primero. Aquellos que se configuran con un número superior de créditos no ven aumentada la docencia destinada a la formación de la competencia digital. Nos mantenemos en una constante que perjudica abiertamente la alfabetización docente. Su presencia es meramente testimonial.

Si medimos su representación en créditos ECTS, tendremos una aproximación más certera a su reconocimiento en los planes de estudio. La presencia de materias tecnológicas no se ofrece de manera equilibrada en los másteres estudiados: desde aquellos que consideran que un crédito (10 horas) es suficiente para su adquisición hasta los que le reconocen un valor superior y le conceden seis créditos, es decir, un total de 60 horas. Estamos ante una oscilación muy llamativa de entre un 10 y un $1.6 \%$ de presencialidad, dato que revela la disparidad existente en las programaciones y la falta de un criterio unificador respecto al valor y la necesidad de este tipo de formación.

Un tanto paradójica y falaz resulta la información mostrada en las competencias, tanto generales como específicas, de los planes de estudio. En ellas se recogen, por ejemplo, la capacidad para utilizar herramientas informáticas y sus aplicaciones en el campo de la investigación, la enseñanza-aprendizaje y la comunicación (Universidad Complutense); conocer y aplicar adecuadamente en el ámbito de la enseñanza del 
español como segunda lengua/lengua extranjera los múltiples recursos que ofrecen las Tecnologías de la Información y la Comunicación (Universidad de León); reflexionar y valorar el papel de las TIC en el aprendizaje (conjunto o autónomo) de una L2, así como sus perspectivas futuras (Universidad de Sevilla); adquirir la capacidad para utilizar las tecnologías de la información y la comunicación en la enseñanza de ELE (Universidad a Distancia de Madrid) o manejar las nuevas tecnologías, recursos informáticos e internet, dirigidas a elaborar materiales para la enseñanza del español y en metodología de enseñanza de ELE (Universidad de Cantabria), por poner algunos ejemplos. Las competencias que se pretenden alcanzar no parecen factibles, vistos los créditos empleados. El uso, además del concepto englobador de TIC, desemboca en una asunción del término a modo de cajón de sastre, donde todo cabe y se ocultan las carencias precisamente por la amplitud del concepto: "Las TIC se han convertido en un reclamo opulento [...] debido principalmente a que no fueron concebidas, en sus orígenes, como herramientas educativas" (ÁLVAREZ, 2017, p. 36). Es este principalmente el origen de la última problemática observada: qué contenidos se han de impartir.

En la mayoría de las asignaturas ofertadas, la principal herramienta que debe ser explotada en el aula es internet, sus recursos y servicios y las implicaciones en docencia e investigación (centrada sobre todo en las búsqueda de materiales y documentos). La visión es sumamente reduccionista, si asumimos las cinco áreas y competencias señaladas en el MCCDD. Hay una querencia colectiva a cubrir el área 1, la relativa a la información y alfabetización informacional, poco o nada se trabaja de las cuatro áreas restantes: comunicación y colaboración, creación de contenidos digitales, seguridad y resolución de problemas. Una competencia digital efectiva pasa obligatoriamente por romper estos límites y abrir las puertas docentes a toda una gama de microhabilidades mucho más necesarias. Todo esto llevaría aparejada una formación superior del docente que redundaría con posterioridad en los alumnos, mejorando también sus competencias y solventando muchas de sus carencias.

\section{Consideraciones finales}

Los contenidos lingüísticos cobran un protagonismo casi tiránico, si los comparamos con las nociones tecnológicas que se recogen en sus planes de estudios. 
Relegadas en fondo y forma, parece no haberse entendido la importancia de la competencia digital docente para una enseñanza eficaz.

La ausencia de contenidos curriculares enfocados a alfabetizar digitalmente a los docentes en los programas de los másteres de ELE revisados en esta investigación demuestra la contradicción en la que vivimos en la sociedad actual; por un lado, se exige una avanzada competencia digital docente, pero, por otro, la falta de asignaturas sobre el funcionamiento, aplicación e implementación de las TIC en el contexto didáctico obliga a que la alfabetización digital se logre de forma autónoma y con la inversión económica y temporal que cada docente de ELE quiera o pueda permitirse.

La revisión bibliográfica destaca la posición central de la competencia digital en las investigaciones actuales sobre innovación didáctica, sin embargo no se alinea a los contenidos curriculares sobre alfabetización digital que ofrecen los másteres de formación de profesorado de ELE. El equilibrio entre el aprendizaje lingüístico y el tecnológico se ve claramente desestabilizado hacia el primero, en consecuencia los docentes que terminan su formación de posgrado presentan unas claras carencias formativas en lo que a alfabetización digital se refiere, hecho que repercute negativamente en la ejecución de su vida profesional tanto en el ámbito educativo como en el investigador y que les lleva a reforzar, ad nauseam, la formación continua docente 4 .

Los resultados de este trabajo indican, en primer lugar, la necesidad de rediseñar los planes curriculares de los programas de máster de ELE para equilibrar la balanza entre la alfabetización académica y digital. Para ello, se pueden crear asignaturas que, por un lado, aseguren la formación de una base sólida de alfabetización digital y asignaturas transversales que combinen la competencia digital con la académica. Existen multitud de publicaciones que recogen de facto esta praxis combinatoria - véase, por ejemplo, Mateos y Alejaldre (2020). En segundo lugar, consideramos que, a pesar de la gran cantidad de investigaciones sobre la importancia de formación tecnológica docente, es necesario diseñar una herramienta de certificación de la competencia digital docente; esta herramienta podría materializarse en un bloque de asignaturas que recogieran los aspectos básicos del MCCDD y cuya superación supusiese la certificación

\footnotetext{
${ }^{4}$ No es nuestra intención evitar dicha formación, todo lo contrario, la creemos vital, como recogen de manera generalizada autores como Junges, Ketzer y Abreu de Oliveira (2018), Ramos Fonseca (2019) o Vieira y Nunes de Faria (2019), entre otros.
}

Educ. Form., Fortaleza, v. 6, n. 1, e3521, jan./abr. 2021

DOI: https://doi.org/10.25053/redufor.v6i1.3521

https://revistas.uece.br/index.php/redufor/index 
de dicha competencia. Serviría asimismo para ampliar la perspectiva en cuanto a lo que conlleva una capacitación digital, y no verla reducida simplemente al uso de internet, al manejo de alguna herramienta digital y poco más. En todo caso y aun inmersos en un mundo fuertemente digitalizado, queda un largo camino que recorrer en el ámbito docente en general y más particularmente en la formación de profesores de español como lengua extranjera.

\section{Referencias}

ALEJALDRE BIEL, L.; ÁLVAREZ RAMOS, E. La competencia digital docente del profesor universitario 3.0. Caracteres: Estudios Culturales y Críticos de la Esfera Digital, Salamanca, v. 8, n. 2, p. 205-236, 2019. Disponible en: https://dialnet.unirioja.es/servlet/articulo?codigo=7323501. Accedido el: 9 jul. 2020.

ÁLVAREZ RAMOS, E. Las TAC al servicio de la formación inicial de maestros en el área de Didáctica de la Lengua y la Literatura: herramientas, usos y problemática. Resed, Cádiz, n. 5, p. 35-48, 2017. Disponible en: https://revistas.uca.es/index.php/resed/article/view/s.1.3n5. Accedido el: 9 jul. 2020.

BADIA, A. Las competencias docentes en el uso formativo de las TIC. In: BADIA, A. (org.). Del docente presencial al docente virtual. Barcelona: UOC, 2004.

BOUDET, J. M. F. Evaluación de la competencia digital docente en la comunidad autónoma de Aragón. Revista Electrónica de Investigación Educativa, Baja California, v. 19 , n. 4 , p. $78-83,2017$.

CARRERA, F. X.; COIDURAS, J. L. Identificación de la competencia digital del profesor universitario: un estudio exploratorio en el ámbito de las ciencias sociales. REDU, Valencia, v. 10, n. 2, p. 273-298, 2012. Disponible en: https://polipapers.upv.es/index.php/redu/article/view/6108. Accedido el: 9 jul. 2020.

COMISIÓN EUROPEA. Competencias clave para el aprendizaje permanente. Recomendación 2006/962/CE del Parlamento Europeo y del Consejo, de 18 de diciembre de 2006, sobre las competencias clave para el aprendizaje permanente. Diario Oficial, L 394, 30 dic. 2006.

COMISIÓN EUROPEA. Competencias clave para el aprendizaje permanente: un marco de referencia europeo. Oficina de Publicaciones Oficiales de las Comunidades Europeas, 2007. Disponible en:

https://www.mecd.gob.es/dctm/ministerio/educacion/mecu/movilidadeuropa/competenciasclave.pdf?documentld=0901e72b80685fb1. Accedido el: 9 jul. 2020. 
DURÁN, M.; GUTIÉRREZ, I.; PRENDES, M. P. Análisis conceptual de modelos de competencia digital del profesorado universitario. Relatec, Extremadura, v. 5, n. 1, p. 97114, 2016a.

DURÁN, M.; GUTIÉRREZ, I.; PRENDES, M. P. Certificación de la Competencia TIC del Profesorado Universitario: diseño y validación de un instrumento. Revista Mexicana de Investigación Educativa, México, DF, v. 21, n. 6, p. 527-556, 2016b.

DURÁN, M.; PRENDES; M. P.; SÁNCHEZ, I. G. Certificación de la Competencia Digital Docente: propuesta para el profesorado universitario. RIED, Madrid, v. 22, n. 1, p. 187205, 2019. Disponible en: http://dx.doi.org/10.5944/ried.22.1.22069. Accedido el: 9 jul. 2020.

ESPAÑA. Real Decreto 1393/2007, de 29 de octubre, por el que se establece la ordenación de las enseñanzas universitarias oficiales. Ministerio de Educación y Ciencia, BOE, n. 260, 30 oct. 2007. Disponible en: https://www.boe.es/buscar/pdf/2007/boe-a2007-18770-consolidado.pdf. Accedido el: 9 jul. 2020.

FERNÁNDEZ, M. C. Los medios audiovisuales, informáticos y nuevas tecnologías en los centros educativos gallegos: presencia y usos. 2001. Tesis - Facultad de Ciencias de la Educación, Santiago de Compostela, 2001.

FERRARI, A. DigComp: A Framework for Developing and Understanding Digital Competence in Europe. Luxembourg: Office of the European Union, 2013. Disponible en: http://publications.jrc.ec.europa.eu/repository/bitstream/JRC83167/lb-na-26035-enn.pdf. Accedido el: 9 jul. 2020.

GABELAS BARROSO, J. A.; MARTA-LAZO, C.; ARANDA, D. ¿Por qué las TRIC y no las TIC? Revista de los Estudios de Ciencias de la Información y de la Comunicación, Madrid, n. 9, s.p., 2012.

GALLEGO, M. J.; GÁMIZ, V.; GUTIÉRREZ, E. El futuro docente ante las competencias en el uso de las tecnologías de la información y comunicación para enseñar. Revista de Tecnología Educativa, Holguín, n. 34, p. 1-18, 2010. Disponible en: https://doi.org/10.21556/edutec.2010.34.418. Accedido el: 9 jul. 2020.

GARCÍA NAVARRO, E.; FERNÁNDEZ MUNÍN, M. C. TIC, TAC, TEP, TRIC. Guix: Elements d'acció educativa, n. 440, p. 78-78, 2017.

GISBERT, M.; ESTEVE, F. Digital learners: La competencia digital de los estudiantes universitarios. La Cuestión Universitaria, Madrid, n. 7, p. 48-59, 2011.

GISBERT, M.; ESPUNY, C.; GONZÁLEZ, J. Incotic: una herramienta para la @utoevaluación diagnóstica de la competencia digital en la universidad. Profesorado: Revista de Currículum y Formación de Profesorado, Granada, v. 15, n. 1, p. 76-89, 2011. 
GUDMUNDSDOTTIR, G. B.; HATLEVIC, O. E. Newly qualified teachers' professional digital competence: implications for teacher education. European Journal of Teacher Education, London, v. 41, n. 2, p. 214-231, 2018.

GUTIÉRREZ, I. Competencias del profesorado universitario en relación al uso de tecnologías de la información y comunicación: análisis de la situación en España y propuesta de un modelo de formación. 2011. Tesis - Universidad Rovira i Virgili, Tarragona, 2011.

GUTIÉRREZ, I.; SERRANO, J. L. Evaluation and development of digital competence in future primary school teachers at the University of Murcia. Journal of New Approaches in Educational Research, Alicante, v. 5, n. 1, p. 51-56, 2016. Disponible en: https://doi.org/10.7821/naer.2016.1.152. Accedido el: 9 jul. 2020.

HANNA, D. Nuevas perspectivas sobre el aprendizaje en la enseñanza universitaria. In: HANNA, D. (ed.). La enseñanza universitaria en la era digital. Barcelona: Octaedro, 2002. p. 59-83.

HATLEVIK, O. E. Examining the relationship between teachers' self- efficacy, their digital competence, strategies to evaluate information and use of ICT at school. Scandinavian Journal of Educational Research, London, v. 61, n. 5, p. 555-567, 2017.

INTEF. Comparativa Marco Competencia Digital Docente (INTEF. Versión octubre, 2017) y Marco Europeo DigCompEdu (JRC-Sevilla, Versión diciembre, 2017). 2017a.

Disponible en: https://intef.es/noticias/comparativa-marco-competencia-digital-docenteintef-version-octubre-2017-y-marco-europeo-digcompedu-jrc-sevilla-version-diciembre2017/. Accedido el: 9 jul. 2020.

INTEF. Marco Común de Competencia Digital Docente. Ministerio de Educación, Cultura y Deporte, 2013. Disponible en: https://www.slideshare.net/educacionlab/marco-cdd-v4. Accedido el: 8 mayo 2020.

INTEF. Marco Común de Competencia Digital Docente. 2017b. Disponible en: http://educalab.es/documents/10180/12809/marco+competencia+digital+docente+2017/a fb07987-1ad6-4b2d-bdc8-58e9faeeccea. Accedido el: 9 jul. 2020.

JUNGES, F. C.; KETZER, C. M.; ABREU DE OLIVEIRA, V. M. Formação continuada de professores: saberes ressignificados e práticas docentes transformadas. Educação \& Formação, Fortaleza, v. 3, n. 9, p. 88-101, 2018. Disponible en: https://revistas.uece.br/index.php/redufor/article/view/858. Accedido el: 9 jul. 2020.

KRUMSVIK, R. Digital competence in Norwegian teacher education and schools. Högre Utbildning, v. 1, n. 1, p. 39-51, 2011. Disponible en:

https://www.researchgate.net/publication/305360830_digital_competence_in_the_norweg ian_teacher_education_and_school. Accedido el: 9 jul. 2020. 
MARCELO, C.; YOT, C.; MAYOR, C. University Teaching with Digital Technologies. Comunicar, Huelva, v. 45, n. 23, p. 117-124, 2015. Disponible en:

https://www.revistacomunicar.com/index.php?contenido=detalles\&numero $=45 \&$ articulo $=4$ 5-2015-12. Accedido el: 9 jul. 2020.

MARTA-LAZO, C. Las TRIC (Tecnologías de la Relación, la Información y la Comunicación) al servicio de la educomunicación para la salud. 2018. Disponible en: http://www.laaab.es/2018/12/las-tric-tecnologias-de-la-relacion-la-informacion-y-lacomunicacion-al-servicio-de-la-educomunicacion-para-la-salud/. Accedido el: 9 jul. 2020.

MARTA-LAZO, C.; GABELAS BARROSO, J. A. Comunicación digital: un modelo basado en el Factor Relacional. Barcelona: UOC, 2016.

MATEOS BLANCO, B.; ALEJALDRE BIEL, L. Microrrelato hipermedial, aprendizaje cognitivo y desarrollo de las destrezas en el marco de ELE. In: CALVO REVILLA, A.; ÁLVAREZ RAMOS, E. (ed.). Microrrelato hipermedial: aproximaciones teóricas y didácticas. Berlín: Peter Lang, 2020. En prensa.

MÉNDEZ SANTOS, M. C.; PANO ALAMÁN, A. Una aproximación al perfil del profesorado de español como lengua extranjera en línea. Revista Internacional de Lenguas Extranjeras, Tarragona, n. 11, p. 1-31, 2019. Disponible en: https://revistes.urv.cat/index.php/rile/article/view/2639. Accedido el: 9 jul. 2020.

MENGUAL, S.; ROIG, R. La enseñanza y las competencias TIC en el contexto universitario. In: SANDOVAL, Y. et al. (coord.). Las tecnologías de la información en los contextos educativos: nuevos escenarios de aprendizaje. Santiago de Cali: Universidad Santiago de Cali, 2012. p. 17-32.

MENGUAL, S.; ROIG, R.; BLASCO, J. Delphi study for the design and validation of a questionnaire about digital competences in higher education. International Journal of Educational Technology in Higher Education, [S.I.], n. 13, p. 1-11, 2016. Disponible en: https://doi.org/10.1186/s41239-016-0009-y. Accedido el: 9 jul. 2020.

MISHRA, P.; KOEHLER, M. J. Introducing Technological Pedagogical Content Knowledge. Annual Meeting of the American Educational Research Association, Nueva York, 2008.

MISHRA, P.; KOEHLER, M. J. Technological pedagogical content knowledge: a framework for teacher knowledge. Teachers College Record, Columbia, v. 108, n. 6, p. 1017-1054, 2006. Disponible en:

http://punya.educ.msu.edu/publications/journal_articles/mishra-koehler-tcr2006.pdf. Accedido el: 9 jul. 2020.

OLCOTT, D.; SCHMIDT, K. La redefinición de políticas y prácticas del profesorado en la era del conocimiento. In: HANNA, D. (Org.). La enseñanza universitaria en la era digital. Barcelona: Octaedro, 2002. p. 265-291.

OLIVEIRA, J. Pre-service teacher education enriched by technology-supported learning environments: a learning technology by design approach. Journal of Literacy and Technology, Boca Raton, v. 11, n. 1, p. 90-109, 2010. 
OLIVEIRA, J.; CERVERA, M.; MARTÍ, M. Learning as representation and representation as learning: a theoretical framework for teacher knowledge in the digital age. Proceedings of World Conference on Educational Multimedia, Hypermedia and Telecommunitcations. Chesapeake: AACE, 2009. p. 2646-2653.

POZOS, K. La competencia digital del profesorado universitario para la sociedad del conocimiento: aproximación a un modelo y validación de un cuestionario de detección de necesidades de formación continua. 2010. Tesis (Doctorado en Calidad y Procesos de Innovación Educativa) - Programa de Posgrado en Calidad y Procesos de Innovación Educativa, Universidad Autónoma de Barcelona, Barcelona, 2010.

PRENDES, M. P. Competencias TIC para la docencia en la universidad pública española: indicadores y propuestas para la definición de buenas prácticas. Informe del proyecto financiado por la Secretaría de Estado de Universidades e Investigación del Ministerio de Educación dentro de la convocatoria Estudio y Análisis (EA2009-0133), 2010. Disponible en: https://www.um.es/competenciastic/. Accedido el: 9 jul. 2020.

PRENDES, M. P.; CASTAÑEDA, L.; PORLÁN, I. G. Competencia para el uso de TIC de los futuros maestros. Comunicar, Huelva, n. 35, p. 175-182, 2010. Disponible en: https://doi.org/10.3916/C35-2010-03-11. Accedido el: 9 jul. 2020.

PRENDES, M. P.; PORLÁN, I. G. Competencias tecnológicas del profesorado en las universidades españolas. Revista de Educación, Madrid, n. 361, p. 196-222, 2013. Disponible en: http://www.educacionyfp.gob.es/dam/jcr:fabfb679-b10c-4f91-b467dc4d8f1e1869/re36108-pdf.pdf. Accedido el: 9 jul. 2020.

PRENDES, M. P.; PORLÁN, I. G.; SÁNCHEZ, F. M. Competencia digital: una necesidad del profesorado universitario en el siglo XXI. RED, Sant'Anna do Livramento, n. 56, p. 222, 2018. Disponible en: http://dx.doi.org/10.6018/red/56/7. Accedido el: 9 jul. 2020.

RAMOS FONSECA, M. G. As tecnologias de informação e comunicação na formação inicial de professores do $1^{\circ}$ ciclo do ensino básico - fatores constrangedores invocados pelos formadores para o uso das tecnologias. Educação \& Formação, Fortaleza, v. 4, n. 11, p. 3-23, 2019. Disponible en: https://revistas.uece.br/index.php/redufor/article/view/254. Accedido el: 9 jul. 2020.

REIG HERNÁNDEZ, D. Disonancia cognitiva y apropiación de las TIC. Revista Telos, Madrid, n. 90, p. 2-2, 2012. Disponible en: https://www.dreig.eu/caparazon/tep-clave-delcambio/. Accedido el: 9 jul. 2020.

ROIG, R.; PASCUAL, A. M. Las competencias digitales de los futuros docentes: un análisis con estudiantes de Magisterio de Educación Infantil de la Universidad de Alicante. @tic: Revista d'Innovació Educativa, Valencia, n. 9, p. 53-60, 2012.

ROMÁN-MENDOZA, E. Aprender a aprender en la era digital: tecnopedagogía crítica para la enseñanza del español LE/L2. Londres: Routledge, 2018. 
TEJADA, J. Competencias docentes. Profesorado: Revista de Currículum y Formación del Profesorado, Granada v. 13, n. 2, p. 1-15, 2009.

TOURÓN, J. et al. Validación de constructo de un instrumento para medir la competencia digital docente de los profesores (CDD). Revista Española de Pedagogía, Madrid, v. 76, n. 269 , p. 25-54, 2018.

UNESCO. Estándares Unesco de competencia en TIC para docentes [versión en español disponible en Eduteka]. 2008. Disponible en:

http://eduteka.icesi.edu.co/articulos/estandaresdocentesunesco. Accedido el: 9 jul. 2020.

UNESCO. Marco de competencias de los docentes en materia de TIC de la Unesco (versión 3), 2019. Disponible en: https://unesdoc.unesco.org/ark:/48223/pf0000371024. Accedido el: 9 jul. 2020.

UNESCO. Unesco ICT Competency Framework for Teachers. 2011. Disponible en: http://unesdoc.unesco.org/images/0021/002134/213475e.pdf. Accedido el: 9 jul. 2020.

VIEIRA, C. E.; NUNES DE FARIA, M. C. Formação de professores nos debates da I Conferência Nacional de Educação (ICNE - 1927), Educação \& Formação, Fortaleza, v. 4, n. 10, p. 95-11, 2019. Disponible en:

https://revistas.uece.br/index.php/redufor/article/view/391. Accedido el: 20 jun. 2020.

Eva Álvarez Ramos, Universidad de Valladolid, Departamento de Enseñanza de la Lengua y la Literatura

i(i) https://orcid.org/0000-0001-7812-6592

Doctora en Filología Hispánica y docente en el Departamento de Didáctica de la Lengua y la Literatura de la Universidad de la Universidad Valladolid. Forma parte del Grupo de Investigación reconocido Literatura Española Contemporánea - Siglos XX y XXI (GIRLEC) de la Universidad de Valladolid y del Grupo de Investigación Reconocido MOVE de la Universidad de Salamanca. Asimismo, es miembro de la Unidad de Investigación Consolidada con área ANEP Literatura Española y Humanidades Digitales. Sus líneas de investigación se centran en Literatura Infantil, Español como Lengua Extranjera, TIC y Enseñanza de Lenguas, Humanidades Digitales aplicadas a la educación y permanencia de la tradición clásica en la poesía española contemporánea.

Contribución de autoría: Se ha hecho cargo más profundamente de la investigación y el trabajo de campo.

E-mail: evamaria.alvarez.ramos@uva.es

\section{Leyre Alejaldre Biel, Columbia University}

ii(i) https://orcid.org/0000-0001-6805-0846

Doctora en Lenguas Modernas, Traducción y Enseñanza de Español como Lengua Extranjera (ELE), máster en Enseñanza de ELE y licenciada en Filología Inglesa. En el año 2000, empezó su carrera docente en el campo de la enseñanza y aprendizaje de ELE y desde entonces ha desempeñado su labor en diversas instituciones en Reino Unido, Estados Unidos, Barbados, Gambia, Tailandia y España a nivel escolar y universitario. En la actualidad desarrolla su labor docente e investigadora en la Universidad de Columbia, donde imparte clases de ELE e investiga sobre el impacto de la implementación de las herramientas digitales en la práctica docente y el aprendizaje de ELE, la influencia del contexto para la inclusión de estas herramientas en el currículo y la necesidad de incluir la alfabetización digital en los planes de estudio.

Contribución de autoría: Se ha ocupado de la fundamentación teórica.

E-mail: la2771@columbia.edu 
Belén Mateos Blanco, Universidad de Valladolid, Departamento de Didática da Língua e Literatura Valladolid

iii@

Licenciada en Periodismo, máster en la Enseñanza de ELE y Estudios Filológicos Superiores y doctora con mención internacional en español: lingüística, literatura y comunicación por la Universidad de Valladolid (UVa) tras realizar su estancia de investigación en la Universidad Nacional Autónoma de México. El título de su tesis Intertextualidad y autorreferencialidad en los microrrelatos del grupo leonés: aplicaciones didácticas para la enseñanza de español como lengua extranjera es una muestra de la confluencia de sus líneas de investigación en las áreas de historia de la literatura, teoría de la literatura y literatura comparada y didáctica. El eclecticismo que define su formación académica le ha permitido desarrollar su carrera profesional en lugares tan dispares como Televisión Castilla y León, el Archivo Provincial de la Diputación de Valladolid o la Fundación Miguel Delibes. Actualmente trabaja como profesora asociada en el Departamento de Didáctica de la Lengua y la Literatura del Campus María Zambrano de la UVa.

Contribución de autoría: Se ha ocupado de la investigación y fundamentación teórica.

E-mail: mariabelen.mateos@uva.es

Editora responsable: Lia Machado Fiuza Fialho Pareceristas ad hoc: Víctor Amar y Álvaro Pérez García

Cómo citar este artículo (ABNT):

ÁLVAREZ RAMOS, Eva; ALEJALDRE BIEL, Leyre; MATEOS BLANCO, Belén. Formación y alfabetización del profesor de ELE: de la competencia lingüística a la tecnológica. Educ. Form., Fortaleza, v. 6, n. 1, e3521, 2020. Disponible en: https://revistas.uece.br/index.php/redufor/article/view/3521

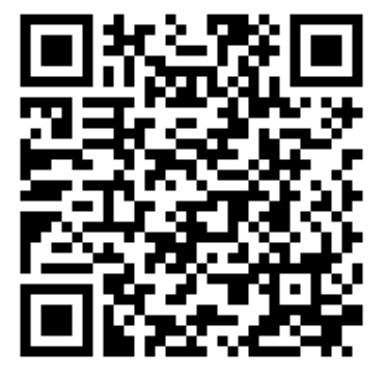

Recibido 11 de julio de 2020.

Aceptado el 18 de agosto de 2020.

Publicado el 13 de noviembre.

Educ. Form., Fortaleza, v. 6, n. 1, e3521, jan./abr. 2021

DOI: https://doi.org/10.25053/redufor.v6i1.3521

https://revistas.uece.br/index.php/redufor/index 